\title{
Idiopathic Isolated Perigastric Omental Panniculitis
}

\author{
Jong Seol Park ${ }^{1}$, Seo Hwa Kim ${ }^{1}$, Byung Wook Yoon', Byoung Su Oh', Dong Sik Yoon ${ }^{1}$, Nurhee Hong ${ }^{2}$, In Kyeom Hwang', \\ Jaehoon Jahng, ${ }^{1,3}$, Yong Sung Kim ${ }^{1,3}$ \\ Departments of Internal Medicine ${ }^{1}$ and Radiology ${ }^{2}$, Wonkwang University Sanbon Hospital, Gunpo, Wonkwang Digestive Disease Research \\ Institute ${ }^{3}$, Wonkwang University School of Medicine, Iksan, Korea
}

\begin{abstract}
Mesenteric panniculitis (MP) is non-specific inflammation of the adipose tissue that primarily involves the small bowel mesentery. Omental involvement has been rarely reported but we report a case of 25 years old woman with isolated lesser omental panniculitis. This patient was diagnosed by CT findings and recovered completely with conservative treatment. Invasive diagnostic methods or surgical exploration has been used to diagnose MP. However, all six reported cases of omental panniculitis including the current case showed a benign course; therefore, awareness of the CT findings is essential for the best diagnosis and management of omental panniculitis. (Korean J Helicobacter Up Gastrointest Res 2015;15:178-181)
\end{abstract}

Key Words: Panniculitis; Omentum; Tomography, x-ray computed; Anti-inflammatory agents, non-steroidal

\section{INTRODUCTION}

Mesenteric panniculitis (MP) is non-specific inflammation of the adipose tissue in the intestinal mesentery. ${ }^{1}$ The precise cause and mechanism of the disease is not fully understood but an underlying malignancy or previous surgery has been suggested as a contributing factor. ${ }^{1,2}$ The symptoms and signs of MP vary from asymptomatic to abdominal pain, anorexia, nausea, pyrexia, diarrhea, weight loss, a palpable mass, rectal bleeding, jaundice, and gastric outlet obstruction. ${ }^{1-3}$

MP primarily involves the small bowel mesentery, which accounts for $90 \%$ of cases $^{4}$ but other areas include the sigmoid mesentery, peripancreatic region, omentum, retroperitoneum, and pelvis. Among these, omental involvement, known as omental panniculitis, is very rare, and only five cases have been reported in the English literature. ${ }^{5-9}$

Invasive methods, such as laparotomy or percutaneous CT-guided biopsy, were performed for the diagnosis in all five cases. However, all five cases showed a benign course and had characteristic CT features. Therefore it is

Received: September 5, 2015 Accepted: September 6, 2015

Corresponding author: Yong Sung Kim

Department of Gastroenterology, Wonkwang University Sanbon Hospital, 327 Sanbon-ro, Gunpo 15865, Korea

Tel: +82-31-390-2975, Fax: +82-31-398-2223, E-mail: wms89@hanmail.net important to understand the CT findings of this condition for a proper diagnosis and to prevent unnecessary invasive procedures. In this report, we present a case of idiopathic isolated perigastric omental panniculitis diagnosed with a CT scan and its complete resolution by non-steroidal anti-inflammatory drug (NSAID) therapy.

\section{CASE REPORT}

A 25 years old female presented with nausea and epigastric pain for the past 3 days. She had no medical history. The epigastric pain was moderate in intensity and was aggravated by motion. The vital signs were stable. A physical examination revealed that the patient was in acute distress and had focal tenderness across the epigastrium on palpation. Laboratory testing, including a complete blood count, liver function tests, and serum electrolytes were within normal ranges; however, a mildly elevated erythrocyte sedimentation rate (ESR) of $25 \mathrm{~cm} / \mathrm{hr}$ was noted. Esophagogastroduodenoscopy showed minimal changes of reflux esophagitis and acute erythematous gastritis. An abdominopelvic CT scan revealed a fat attenuated lesion around the gastric lesser curvature with peri-lesional infiltration (Fig. 1). Based on these CT imaging features, she was diagnosed with isolated-omental panniculitis around the gastric lesser curvature side. We performed additional tests, such as anti-nuclear antigen,

Copyright $\odot 2015$ Korean College of Helicobacter and Upper Gastrointestinal Research

() The Korean Journal of Helicobacter and Upper Gastrointestinal Research is an Open-Access Journal. All articles are distributed under the terms of the Creative Commons Attribution Non-Commercial License (http://creativecommons.org/licenses/by-nc/4.0) which permits unrestricted non-commercial use, distribution, and reproduction in any medium, provided the original work is properly cited. 


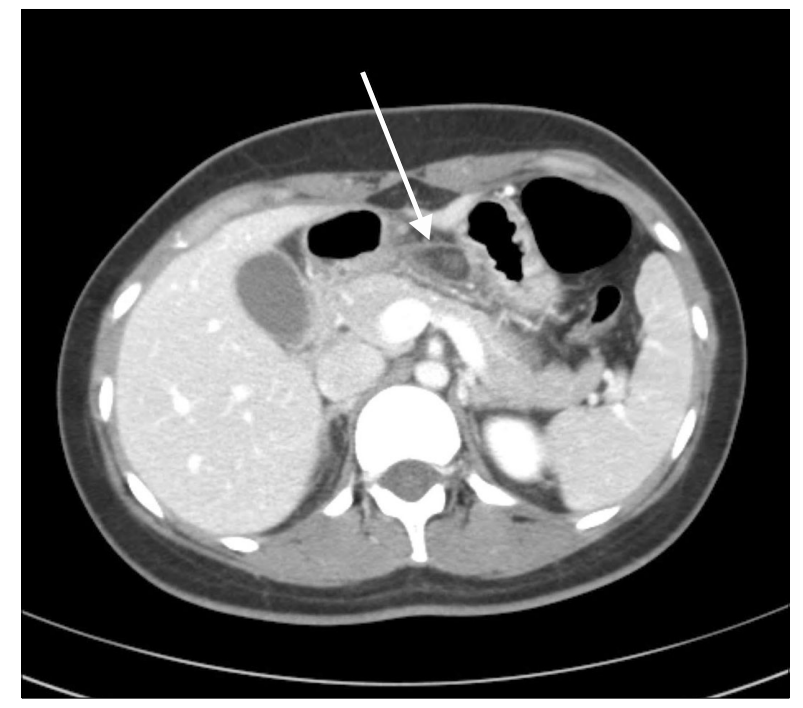

Fig. 1. Contrast-enhanced abdominal CT scan shows a fat attenuated lesion with perilesional infiltration around the gastric lesser curvature (white arrow).

rheumatoid factor, lupus anti-coagulant, and coagulation factors, to determine the underlying cause, but all results were within normal limits. The patient was started on $200 \mathrm{mg}$ ibuprofen three times/day. Her epigastric pain and nausea decreased gradually in frequency and intensity. The patient's pain disappeared completely within 4 weeks. A 2-month follow-up CT scan revealed no previous fatty infiltrative lesion (Fig. 2). Thus, no further treatment was necessary.

\section{DISCUSSION}

The prevalence of MP in earlier studies was $0.6 \%,{ }^{1}$ but use of CT imaging has increased and more recent studies have reported a prevalence as high as $7.83 \%{ }^{10} \mathrm{MP}$ rarely involves the omentum. Five cases have been reported; three involved the greater omentum, ${ }^{6,8}$ one involved both the greater omentum and mesentery, and one involved the lesser omentum. ${ }^{5}$ Four of these cases were diagnosed during a laparotomy ${ }^{5-8}$ and one by percutaneous CT-guided biopsy.? Three cases underwent surgical resection $^{5,6,8}$ and the others received conservative treatment. $^{7,9}$

The precise cause of MP is unknown. ${ }^{1}$ Possible causes of the disease include malignancy, autoimmune disease,

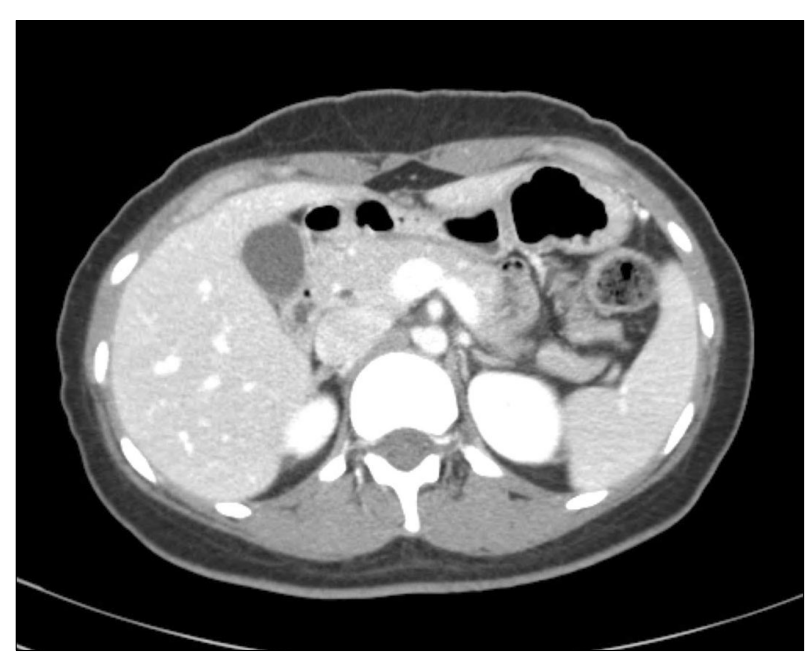

Fig. 2. A 2-month follow-up contrast-enhanced abdominal CT scan shows that the previous fatty lesion between the pancreas body, gastric lesser curvature, and left hepatic lobe had disappeared.

infection, drugs, mesenteric thrombosis, mesenteric arteriopathy, thermal or chemical injuries, vasculitis, abdominal surgery, pancreatitis, urine or bile leakage, gall stones, cirrhosis, abdominal aortic aneurysm, peptic ulcer, and hypersensitivity reactions. ${ }^{1,3}$

An association between MP and intra-abdominal malignancy had been suggested in some studies. ${ }^{1,10}$ In contrast, one prospective study investigating 613 patients imaged with abdominal multi-detector (MD) CT reported that the general prevalence of MP does not significantly differ in "neoplastic" and "non-neoplastic" groups of patients. They concluded that the value of MP in terms of predicting an associated neoplasm is probably not relevant. ${ }^{10}$ However, clinicians should consider the possibility of an intra-abdominal malignancy in a case of MP. All six cases of omental panniculitis, including our case, were not related to a neoplastic condition.

The symptoms and signs of MP vary from asymptomatic to abdominal pain, anorexia, nausea, pyrexia, diarrhea, weight loss, a palpable mass, rectal bleeding, jaundice, and gastric outlet obstruction. ${ }^{2,3,11}$ Among all six cases including the present case, five were idiopathic with a short history of symptoms over $3 \sim 7$ days. ${ }^{5,6,8,9}$ The sixth case was caused by a delayed-type hypersensitivity reaction due to paroxetin and had a 4-month history of various symptoms including weight loss. ${ }^{7}$ Laboratory find- 
ings are generally nonspecific in patients with MP but an elevated ESR, neutrophilia, and anemia have been observed. $^{12,13}$ Patients with MP have a benign course, and follow-up studies have shown good stability of the CT findings in about $85 \%$ of MP cases. ${ }^{10,14}$

$\mathrm{CT}$ findings of MP can provide clues for diagnosing the disease. Coulier described typical signs of MP on CT as a well-defined "mass effect" on neighboring structures, mesenteric fat tissue of inhomogeneous higher attenuation than adjacent abdominal fat, small soft tissue nodules, nodules surrounded by a hypoattenuated fatty halo (halo sign), a hyperattenuating pseudocapsule surrounding the mesenteric fat tissue. ${ }^{10}$ The last two signs are not constant but are very specific for the MP diagnosis and are not usually reported for other mesenteric diseases, such as lymphoma, liposarcoma, lipoma, or mesenteric carcinomatosis. ${ }^{1,10,15}$

Omental panniculitis has no specific imaging findings compared with those of MP, except the location. Among the six cases, including our case, five patients presented with a high attenuation fatty lesion compatible with the diagnosis of omental panniculitis. Only one case of a drug hypersensitivity reaction was manifested as diffuse omental and mesenteric fat infiltration due to massive infiltrates composed predominantly of eosinophils and mononuclear cells along with evolving fibrosis. ${ }^{7}$

MP has been confirmed previously by a pathological examination. However, recent technical advances in MDCT distinguish MP from mesenteric tumoral involvement. In addition, positron emission tomography/CT enhances diagnostic accuracy to discriminate malignancies. ${ }^{10}$ If a CT scan presents features, such as soft nodules $>10 \mathrm{~mm}$ in diameter, retroperitoneal extension, vessel displacement, invasion of the bowel, or an increase in the size of the nodules on a follow-up CT, a biopsy should be performed to exclude malignancy. ${ }^{16}$

In our case, panniculitis was diagnosed based on CT findings. Although the peri-gastric location of the lesion was unusual, a biopsy was not performed because no evidence of intra-abdominal malignancy was detected on CT or endoscopy. The lesion disappeared completely on a follow-up CT after NSAID treatment.

Treatment for MP is considered in symptomatic patients, but no consensus exists for the treatment. ${ }^{14}$ Various medications, including steroids, colchicine, azathioprine, tamoxifen, antibiotics, anti-emetics, or radiotherapy can be beneficial ${ }^{17,18}$ A surgical option can be considered if medical therapy fails to achieve pain relief or in cases of an inflammatory bowel obstruction or perforation. ${ }^{19}$ If a young patient with MP has no vasculitis and has no evidence of malignancy on a CT scan, such as in our case, conservative treatment may be sufficient.

To conclude, despite the low prevalence, omental panniculitis can be diagnosed based on characteristic CT features. Considering the benign nature of omental panniculitis, awareness of CT findings is essential for an optimal diagnosis and preventing an unnecessary biopsy.

\section{REFERENCES}

1. Daskalogiannaki M, Voloudaki A, Prassopoulos P, et al. CT evaluation of mesenteric panniculitis: prevalence and associated diseases. AJR Am J Roentgenol 2000;174:427-431.

2. Cuff R, Landercasper J, Schlack S. Sclerosing mesenteritis. Surgery 2001;129:509-510.

3. Coulier B. Mesenteric panniculitis. Part 1: MDCT--pictorial review. JBR-BTR 2011;94:229-240.

4. McCrystal DJ, O'Loughlin BS, Samaratunga H. Mesenteric panniculitis: a mimic of malignancy. Aust N Z J Surg 1998;68: 237-239.

5. Katz ME, Heiken JP, Glazer HS, Lee JK. Intraabdominal panniculitis: clinical, radiographic, and CT features. AJR Am J Roentgenol 1985;145:293-296.

6. Lheureux P, Matos C, Charlier PH, et al. Omental panniculitis: an unusual cause of acute appendiceal syndrome. Ann Emerg Med 1987;16:224-226.

7. Rozin A, Bishara B, Ben-Izhak O, Fischer D, Carter A, Edoute Y. Fibrosing omental panniculitis and polyserositis associated with long-term treatment by paroxetine. Isr Med Assoc J 2000;2:714-716.

8. Hirono S, Sakaguchi S, Iwakura S, Masaki K, Tsuhada K, Yamaue H. Idiopathic isolated omental panniculitis. J Clin Gastroenterol 2005;39:79-80.

9. Jeon EJ, Cho SM. Idiopathic isolated omental panniculitis confirmed by percutaneous CT-guided biopsy. Gut Liver 2009;3: 321-324.

10. Coulier B. Mesenteric panniculitis. Part 2: prevalence and natural course: MDCT prospective study. JBR-BTR 2011;94:241-246.

11. Durst AL, Yarom R, Luttwak EM. Malignant fibromatous peritoneal mesothelioma associated with liposclerotic mesenteritis. Am J Gastroenterol 1971;55:477-481.

12. Emory TS, Monihan JM, Carr NJ, Sobin LH. Sclerosing mesenter- 
itis, mesenteric panniculitis and mesenteric lipodystrophy: a single entity? Am J Surg Pathol 1997;21:392-398.

13. Durst AL, Freund H, Rosenmann E, Birnbaum D. Mesenteric panniculitis: review of the leterature and presentation of cases. Surgery 1977;81:203-211.

14. Sabaté JM, Torrubia S, Maideu J, Franquet T, Monill JM, Pérez C. Sclerosing mesenteritis: imaging findings in 17 patients. AJR Am J Roentgenol 1999;172:625-629.

15. van Breda Vriesman AC, Schuttevaer HM, Coerkamp EG, Puylaert JB. Mesenteric panniculitis: US and CT features. Eur Radiol 2004; 14:2242-2248.

16. Wat SY, Harish S, Winterbottom A, Choudhary AK, Freeman AH.
The CT appearances of sclerosing mesenteritis and associated diseases. Clin Radiol 2006;61:652-658.

17. Mazure R, Fernandez Marty P, Niveloni S, et al. Successful treatment of retractile mesenteritis with oral progesterone. Gastroenterology 1998;114:1313-1317.

18. Miyake H, Sano T, Kamiya J, et al. Successful steroid therapy for postoperative mesenteric panniculitis. Surgery 2003;133:118119.

19. Gu GL, Wang SL, Wei XM, Ren L, Li DC, Zou FX. Sclerosing mesenteritis as a rare cause of abdominal pain and intraabdominal mass: a cases report and review of the literature. Cases J 2008;1:242. 